\title{
Phase-coherent multilevel two-photon transitions in cold Rb atoms: Ultrahigh-resolution spectroscopy via frequency-stabilized femtosecond laser
}

\author{
Tai Hyun Yoon,* Adela Marian, John L. Hall, and Jun Ye ${ }^{\dagger}$ \\ JILA, National Institute of Standards and Technology, and University of Colorado, Boulder, Colorado 80309-0440
}

(Received 20 July 2000; published 6 December 2000)

\begin{abstract}
A two-photon transition in cold $\mathrm{Rb}$ atoms will be probed with a phase-coherent wide bandwidth femtosecond laser comb. Frequency domain analysis yields a high-resolution picture where phase coherence among various transition pathways through different intermediate states produces interference effects on the resonantly enhanced transition probability. This result is supported by the time domain Ramsey interference effect. The two-photon transition spectrum is analyzed in terms of the pulse repetition rate and carrier frequency offset, leading to a cold-atom-based frequency stabilization scheme for both degrees of freedom of the femtosecond laser.
\end{abstract}

DOI: 10.1103/PhysRevA.63.011402

PACS number(s): 32.80.Qk, 32.80.-t, 42.65.Re

The recent rapid progress in the generation of wide bandwidth optical combs based on Kerr-lens mode-locked femtosecond (fs) lasers have created many dramatic possibilities. The field of optical frequency metrology has been revolutionized with the capability of a single-step phase-coherent frequency bridge across several hundred $\mathrm{THz}[1,2]$, leading to precision optical frequency measurements $[1,2]$ and a direct link between optical and microwave standards [2]. For the ultrafast science, the recent work on stabilization of the relative phase between the pulse envelope and the optical carrier [3] should lead to more exquisite control of the pulse shape and timing, opening the door for many interesting experiments in the area of extreme nonlinear optics and coherent control.

Such a remarkable measurement capability has arrived at a time when optical frequency standards based on a single ion or cold atoms are emerging as potentially the most stable clocks of any sort [4]. Although not an ultimate choice for an optical clock system, the two-photon transition of Rb atoms at $778 \mathrm{~nm}$ presents an attractive alternative [5]. However, our motivation to study the Rb two-photon transition with a frequency comb generated from a fs laser (fs comb) has a much broader reach than the mere improvement of the current $\mathrm{cw}-$ laser based two-photon system. In this Rapid Communication we will show how a phase-coherent wide-bandwidth optical comb induces the desired multipath quantum interference effect for a resonantly enhanced two-photon transition rate. The analysis is carried out in both the frequency and the time domains to illustrate the novel aspect of phase-coherent pulses with a wide bandwidth that covers all the relevant intermediate states. We will discuss the consequence of these results in terms of the absolute control of both degrees of freedom of the fs comb, namely the comb spacing and the carrier offset frequency. The multipulse interference in the time domain gives an interesting variation and generalization of the two-pulse based temporal coherent control of the excited-state wave packet [6].

\footnotetext{
*Permanent address: KRISS, 1 Toryong, Yusong, Taejon 305600 , Korea.

${ }^{\dagger}$ Electronic address: Ye@jila.colorado.edu
}

Doppler-free two-photon spectroscopy is carried out usually with two equal-frequency cw-laser beams propagating in opposite directions [7]. The two-photon transition rate can be resonantly enhanced via the intermediate states with two different laser frequencies [8] or accelerated atomic beams [9], with a small residual Doppler effect. High-resolution Ramsey-type two-photon spectroscopy using pulsed light has also been demonstrated [10], with the recent extension to the cold atoms [11]. The unique feature of the present work is that the wide bandwidth optical comb allows all relevant intermediate states to resonantly participate in the twophoton excitation process, permitting the phase coherence among different comb components to induce a stronger transition rate through quantum interference.

Figure 1(a) shows the relevant ${ }^{87} \mathrm{Rb}$ energy levels involved in the two-photon transition from the ground state $5 S_{1 / 2}$ to the excited state $5 D_{3 / 2}$. The dipole-allowed intermediate states, $5 P_{3 / 2}$ and $5 P_{1 / 2}$, lie $\sim 2 \mathrm{~nm}$ and $17 \mathrm{~nm}$ below the virtual level, respectively. Also shown is a regularly spaced comb of optical frequencies around $800 \mathrm{~nm}$. The comb frequency spacing $(\Delta)$ is equal to the inverse of the pulse round-trip time $(T)$ inside the cavity. The uniformity of $\Delta$ has been demonstrated at a level of $1 \times 10^{-17}$ [1]. The

(a)

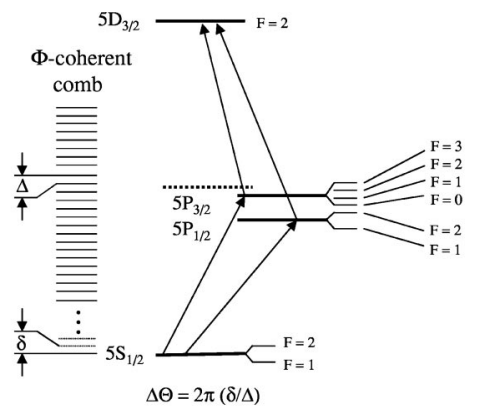

(b)

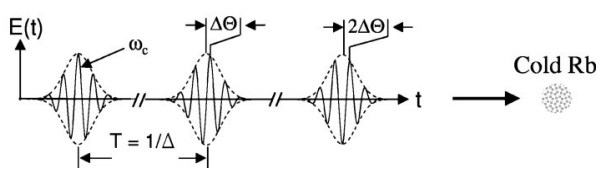

FIG. 1. (a) Schematic of the relevant energy levels of the ${ }^{87} \mathrm{Rb}$ atom. (b) Sequence of mode-locked pulses. The carrier-envelope phase shift $\Delta \Theta$ is shown. 
frequency of any comb line can thus be expressed as an integer multiple of $\Delta$ plus an offset frequency $\delta$ that arises from a difference in phase $\left(v_{p}\right)$ and group $\left(v_{g}\right)$ velocities of the pulses in the laser cavity. A 10-fs laser has a sufficient bandwidth to have comb components line up with corresponding hyperfine states of $5 P_{3 / 2}$ and $5 P_{1 / 2}$ to resonantly enhance the two-photon transition. This multipath quantum interference can be controlled by the tuning of mode spacing and carrier offset frequency, leading to a scheme of simultaneous stabilization of both $\Delta$ and $\delta$, and thereby the entire comb. The frequency domain analysis is complemented perfectly by the time domain multipulse Ramsey interference picture, as illustrated in Fig. 1(b), where the carrier-envelope phase shift $\Delta \theta$ is also shown.

To study the dramatic enhancement of the two-photon transition rate $\Gamma_{g f}$ by the resonant intermediate states and the subsequent interference among different paths, we calculate $\Gamma_{g f}$ analytically in both frequency and time domains using the time-dependent second-order perturbation theory. The perturbative part of the Hamiltonian is $H_{I}=-\mu E(t)$, where $\mu$ is the electric dipole moment and $E(t)$ is the applied electric field. For the transition of $5 S_{1 / 2} F^{\prime \prime}=2 \rightarrow 5 D_{3 / 2} F=2$, we have five participating intermediate states, namely, $5 P_{1 / 2} F^{\prime}$ $=1,2$ and $5 P_{3 / 2} F^{\prime}=1,2,3$. We denote $\left|\psi_{g}\right\rangle,\left|\psi_{m}\right\rangle \quad(m=1$ -5 ), and $\left|\psi_{f}\right\rangle$ as the ground state, five intermediate states, and the final excited state, respectively. $\mu_{1}$ and $\mu_{2}$ are the dipole moments associated with $\left|\psi_{g}\right\rangle \rightarrow\left|\psi_{m}\right\rangle$ and $\left|\psi_{m}\right\rangle$ $\rightarrow\left|\psi_{f}\right\rangle$. We note in passing that, for the case of polarizationgradient cooled $\mathrm{Rb}$ atoms, the first-order Doppler shift can be neglected, since its magnitude $(\sim 100 \mathrm{kHz})$ is much smaller than the natural linewidths of the intermediate states $(\sim 6 \mathrm{MHz})$.

For the frequency domain analysis, we express the spectrum of the electric field $E(t)$ of the fs comb as

$$
E(t)=\frac{1}{2} \sum_{n} E_{n} \exp \left[-i\left(\omega_{r}+n 2 \pi \Delta\right) t\right]+\text { c.c. }
$$

where $\omega_{r}=2 \pi\left(N_{r} \Delta+\delta\right)$ is a reference frequency, and $N_{r}$ is an integer. The atomic wave function can be expressed as $|\Psi(t)\rangle=\Sigma_{m} C_{m}(t)\left|\psi_{m}\right\rangle \exp \left(-i E_{m} t / \hbar\right)$ in terms of the slowly varying probability amplitudes $C_{m}(t)$ of atomic states $\left|\psi_{m}\right\rangle$ of energy $E_{m}$, with $m$ covering all relevant states. We set $C_{m}(t)=C_{m}^{(0)}(t)+C_{m}^{(1)}(t)+C_{m}^{(2)}(t)+\cdots$, with the initial condition $C_{m}^{(0)}(0)=\delta_{m g}$. The interaction between the atoms and the electric field induces

$$
\begin{aligned}
& \left(d / d t+\pi \gamma_{m}\right) C_{m}^{(k+1)} \\
& \quad=-i / \hbar \sum_{n}\left\langle\psi_{m}\left|H_{I}\right| \psi_{n}\right\rangle \exp \left(i \omega_{n m} t\right) C_{m}^{(k)},
\end{aligned}
$$

where $\omega_{n m}=\left(E_{m}-E_{n}\right) / \hbar$ is the frequency of the transition $\left|\psi_{n}\right\rangle \rightarrow\left|\psi_{m}\right\rangle . \quad \gamma_{m}$ are the corresponding decay terms. The second-order excited-state probability amplitude $C_{f}^{(2)}(t)$ can be obtained as

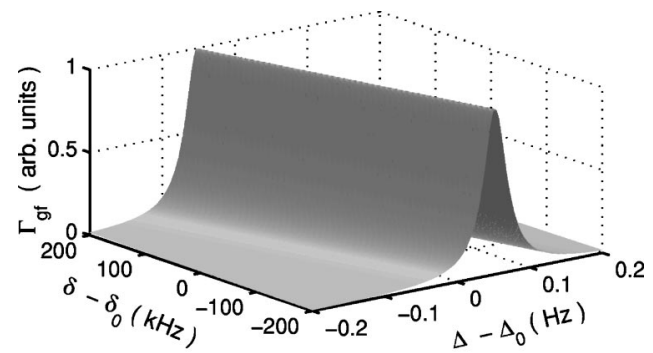

(a)

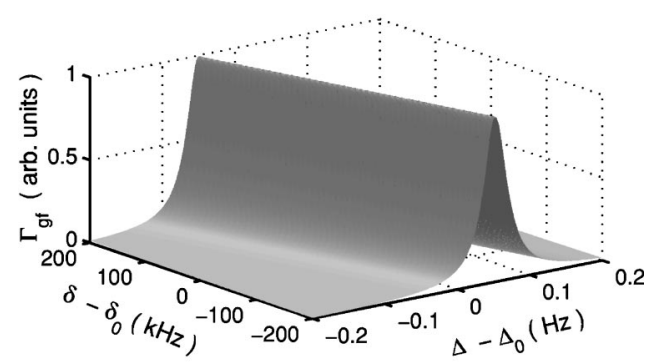

(b)

FIG. 2. $\Gamma_{g f}$ as a function of $\Delta$ and $\delta$, calculated from the analytical expressions obtained in frequency domain (a) and in time domain (b). The values of $\Delta_{0}$ and $\delta_{0}$ are $100 \mathrm{MHz}$ and $15.69 \mathrm{MHz}$, respectively, for both (a) and (b). The pulse width is 10 fs for (b).

$$
\begin{aligned}
C_{f}^{(2)}(t)= & 5 \sum_{p} \sum_{q} \frac{\exp \left\{-i\left[\omega_{g f}-(p+q) 2 \pi \Delta-4 \pi \delta\right] t\right\}}{i\left[\omega_{g f}-(p+q) 2 \pi \Delta-4 \pi \delta\right]+\pi \gamma_{f}} \\
& \times \sum_{m} \frac{\beta_{1} \beta_{2}}{i\left[\omega_{g m}-2 \pi(p \Delta+\delta)\right]+\pi \gamma_{m}}
\end{aligned}
$$

where $\beta_{1(2)}=\mu_{1(2)} E_{0} / 2 \hbar$ is the Rabi frequency associated with the transition $\left|\psi_{g}\right\rangle \rightarrow\left|\psi_{m}\right\rangle \quad\left(\left|\psi_{m}\right\rangle \rightarrow\left|\psi_{f}\right\rangle\right)$. The twophoton transition rate is $\Gamma_{g f}=\gamma_{f}\left|C_{f}^{(2)}(t)\right|^{2}$, where $\gamma_{f}$ is the natural linewidth of the excited state $\left|\psi_{f}\right\rangle$. $\Gamma_{g f}$ has two resonance denominators. One originates from energy conservation of the two-photon transition where the sum of the two comb frequencies matches $\omega_{g f}$; the other results from the single-photon resonance of $\left|\psi_{g}\right\rangle \rightarrow\left|\psi_{m}\right\rangle$. The linewidths of the two resonances are $\gamma_{f}$ and $\gamma_{m}$, respectively. Each intermediate state provides a resonant pathway and they add coherently to yield the total transition rate $\Gamma_{g f}$, owing to the fact that different comb components are phase coherent.

In the time domain, the train of $(N+1)$ mode-locked pulses can be represented by

$$
\begin{aligned}
E(t)= & \sum_{l=0}^{N} E_{l} \exp \left[-(t-l T)^{2} / 2 \tau^{2}\right] \\
& \times \exp \left[-i \omega_{c}(t-l T)-i l \Delta \Theta\right],
\end{aligned}
$$

with $\Delta \Theta=\omega_{c} T\left(1-v_{g} / v_{p}\right)=2 \pi \delta / \Delta+n 2 \pi$. Here $\omega_{c}$ is the carrier frequency, and $l$ and $n$ are integers. During the time periods of $l T-\tau / 2$ to $l T+\tau / 2$, one pulse, with its sufficiently wide bandwidth, drives the probability amplitudes of all intermediate states $C_{m}^{(1)}(t)$ with the first-order perturbation and of the final state $C_{f}^{(2)}(t)$ with the second-order perturbation. In between two pulses, the atomic states evolve freely ac- 
cording to the unperturbed Hamiltonian $H_{0}$ along with the appropriate decay rates. The next pulse, with the corresponding phase shifts, will continue to build the atomic probability amplitudes in a coherent fashion. This process will of course reach a state of equilibrium since the excited state has a lifetime of $1 /\left(2 \pi \gamma_{f}\right)$. To reach an analytical solution, we simplify the pulse shape to be square. After $N+1$ pulses, the probability amplitude $C_{f}^{(2)}(t)$ is

$$
\begin{aligned}
C_{f}^{(2)}(N T)= & C_{f}^{(2)}(\tau) \sum_{l=0}^{N} e^{-\pi \gamma_{f}(N-l) T} e^{i\left(\omega_{g f}-4 \pi \delta\right) l T} \\
& +\sum_{m=1}^{5} \beta_{2} S^{\tau}(m f) \sum_{l=1}^{N} C_{m}(l T) \\
& \times e^{-\pi \gamma_{f}(N-l) T} e^{i\left(\omega_{m f}-2 \pi \delta\right) l T},
\end{aligned}
$$

with

$$
\begin{aligned}
& C_{f}^{(2)}(\tau)=\sum_{m=1}^{5} \beta_{1} \beta_{2}\left[S^{\tau}(g f)-S^{\tau}(m f)\right] \frac{1}{\omega_{g m}-\omega_{c}}, \\
& C_{m}(l T)=\beta_{1} S^{\tau}(g m) \sum_{n=0}^{l-1} e^{-\pi \gamma_{m}(l-n) T} e^{i\left(\omega_{g m}-2 \pi \delta\right) n T},
\end{aligned}
$$

where

$$
S^{\tau}(v)=i \frac{\sin \left[\left(\omega_{v}-s \omega_{c}\right) \tau / 2\right]}{\left(\omega_{v}-s \omega_{c}\right) / 2}
$$

In the last expression, $s=1$ for $v=g m, m f$ and $s=2$ for $v$ $=g f$. It is interesting to note that the phase terms in Eq. (4) depend explicitly on the carrier-envelope phase shift $\Delta \Theta$ $(=2 \pi \delta / \Delta+n 2 \pi)$, but not on the carrier frequency $\omega_{c}$, which has an effect only on the relative signal size.

The measured values of the $\mathrm{Rb}$ transition frequencies, hyperfine intervals, and decay rates are used in the present calculation [12]. Figure 2 shows a typical $\Gamma_{g f}$ as a function of $\Delta$ and $\delta$, with curve (a) calculated from the frequency domain [Eq. (2)] and (b) calculated from the time domain [Eq. (4)]. In the frequency domain calculation, the frequency coverage of the comb lines used in the calculation far exceeds the hyperfine splittings among the intermediate states. Also the number of pulses used for the time domain calculation is larger than necessary to reach the steady state. (See Fig. 3.) The agreement between the two approaches is perfect, with both graphs generated around the same nominal values of $\Delta_{0}=100 \mathrm{MHz}$ and $\delta_{0}=15.69 \mathrm{MHz}$. The resonance width associated with $\Delta$ (with a fixed $\delta$ ) is determined primarily from the two-photon resonance condition and is on the order of $\gamma_{f} /\left(\omega_{g f} / 2 \pi \Delta\right)$. The resonance width associated with $\delta$ is roughly on the order of $\gamma_{f}(300 \mathrm{kHz})$.

To show the effect of multicomb line contributions, we plot in Figs. 3(a) and 3(b) the growth of $\Gamma_{g f}$ vs the number of participating comb lines. The calculation is performed in the frequency domain. Figure 3(a) shows a hypothetical case where we use only one intermediate state to avoid quantum interference. We can see that the main contribution to the
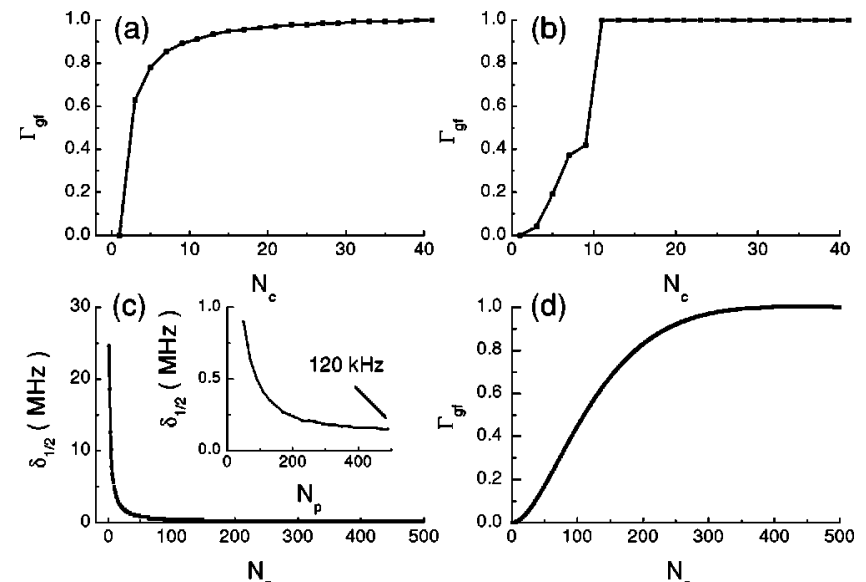

FIG. 3. (a) $\Gamma_{g f}$ vs the number of comb lines $N_{c}$ calculated in the frequency domain. Only one intermediate state is considered. (b) Same as (a), except all five intermediate states are included. (c) Dependence of the two-photon transition linewidth $\delta_{1 / 2}$ on pulse number $\left(N_{p}\right)$. (d) $\Gamma_{g f}$ vs pulse number $\left(N_{p}\right)$.

signal comes from the first comb pair with one component tuned near $\left|\psi_{g}\right\rangle \rightarrow\left|\psi_{m}\right\rangle$ and the other tuned near $\left|\psi_{m}\right\rangle$ $\rightarrow\left|\psi_{f}\right\rangle$. This is understandable considering that the comb spacing $\Delta(\sim 100 \mathrm{MHz})$ is much larger than $\gamma_{m}(5 \mathrm{MHz})$. When we include all five intermediate states, the "saturation" curve shown in Fig. 3(b) is less smooth, with the interference among different pathways contributing to a sudden change of the signal size. Still, only about ten comb lines need to be included for the final signal size. Figures 3(c) and 3(d) illustrate the evolution of the time domain interference effect as we plot the resonance linewidth and size with respect to the increasing pulse numbers. Clearly the Ramsey interference enhances the frequency resolution as more pulses participate, with the final linewidth limited basically by $\gamma_{f}$ itself [Fig. 3(c) and its inset], after 200 pulses or so. The signal size also reaches a stable value after a similar number of pulses [Fig. 3(d)]. The number of pulses needed to reach equilibrium is on the order of the ratio of the excited state lifetime over the pulse repetition period $T$. The bandwidth issue of the comb can be explored with the original Gaussian pulse shape in Eq. (3). We find that for a pulse of constant energy, the signal size remains unchanged when the pulse width $\tau$ increases, till about $30 \mathrm{fs}$, where the bandwidth becomes too small to cover the intermediate states and the signal size starts to decrease. In the calculation for Fig. 2, $\tau$ is 10 fs.

While the results shown in Fig. 2 are informative, they are hardly useful for frequency control of the fs comb. Clearly, Fig. 2 provides only one constraint for both $\Delta$ and $\delta$ and therefore we will not be able to control them independently. The reason lies in the fact that we have chosen the zero frequency as the reference point for both degrees of freedom associated with the comb. In other words, the effects on the comb frequency by the changes of $\Delta$ and $\delta$ are too similar and so an orthogonalized control is difficult. This situation can be remedied in practice. Optically one can adjust the laser cavity such that the frequency $\omega_{p}$ at which the cavity dispersion is not sensitive (to first order) to a rotating mirror lies above $\omega_{g f} / 2$. In this case the frequencies of the comb 
components interacting with the intermediate states will be shifted down with an increasing $\Delta$, but up with an increasing $\delta$, leading to a possible orthogonal control. Another approach is to use electronic means to mix the control information for both $\Delta$ and $\delta$ such that two new orthogonal signals can be generated. Mathematically, this orthogonalization process amounts to a change of variables in Eq. (2), which we now rewrite as

$$
\begin{aligned}
C_{f}^{(2)}(t)= & \frac{5}{2 \pi} \sum_{k} \frac{\exp \left\{-4 \pi i \delta_{1} t\right\}}{i\left(-2 \delta_{1}\right)+\gamma_{f} / 2} \\
& \times \sum_{m} \frac{\beta_{1} \beta_{2}}{i\left[\omega_{g m}-\omega_{g f} / 2-2 \pi\left(k \Delta+\delta_{1}\right)\right]+\pi \gamma_{m}}
\end{aligned}
$$

where $\delta_{1}=\delta-\bmod \left(\omega_{g f} / 4 \pi \Delta\right)$. Figure 4 displays the resonance picture against $\delta_{1}$ and $\Delta$. We choose to show two representative cases with $\Delta_{0}=101.851871 \mathrm{MHz}$ for (a) and $\Delta_{0}=105 \mathrm{MHz}$ for (b). Specific values of $\Delta_{0}$ are sought to have corresponding comb components tuned near a majority of the five intermediate states. Furthermore, to have a maximum enhanced peak, detunings between the five intermediate states and their respective comb lines should all have the same sign. The single peak in (a) shows an enhanced $\Gamma_{g f}$ (by $3^{2}=9$ times, compared against a single-state resonance) due to three constructively interfering states. Fig. 4(b) shows a situation where no comb lines are tuned near the resonances of intermediate states and yet constructive interference still helps to enhance the signal. The resonance width associated with $\Delta$ is on the order of $\gamma_{m} /\left[\left(\omega_{g f} / 2-\omega_{g m}\right) / 2 \pi \Delta\right]$. Simultaneous control of both $\Delta$ and $\delta$ is now clearly feasible. Atom-based frequency stabilization of a fs laser provides long-term stability and should be an attractive complement to other approaches, including the locking of $\Delta$ to a microwave source [1,2], the locking of a fs laser to an optical cavity [13], self-referenced $f-2 f$ heterodyne locking [3], and a fs comb phase locked to an ultrastable cw laser [14].

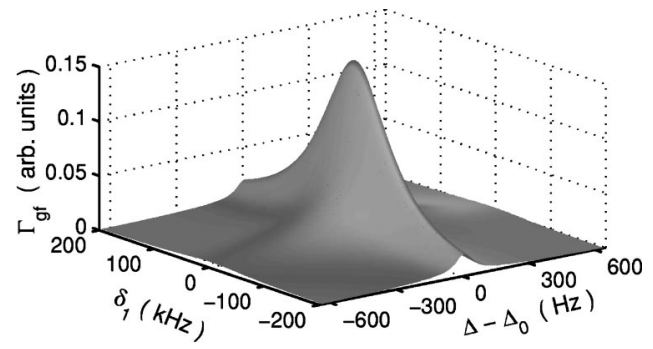

(a)

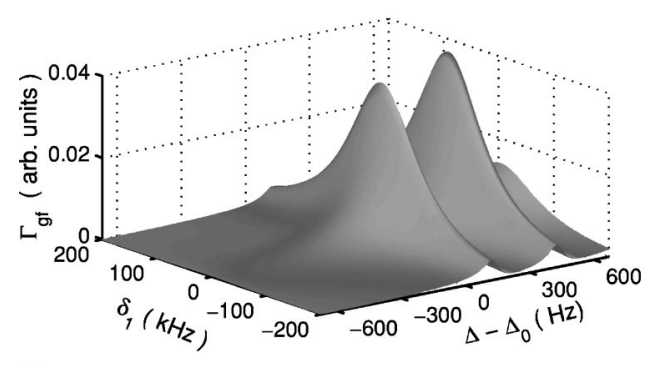

(b)

FIG. 4. $\Gamma_{g f}$ as functions of $\Delta$ and $\delta_{1}$ [Eq. (5)]. All parameters are the same as in Fig. 2, except $\Delta_{0}=101.851871 \mathrm{MHz}$ for (a) and $\Delta_{0}=105 \mathrm{MHz}$ for (b).

In summary, we show that the two-photon process can be dramatically enhanced through the use of a phase-coherent fs comb resonantly exciting stepwise transitions. Quantum interference among different pathways leads to the desired information of the atomic structure, while providing an absolute reference for a complete control of the fs laser. The ultrahigh-resolution aspect of this approach can be understood also from the time domain analysis where a series of Ramsey-type atom-pulse interactions provide a long coherent interrogation time.

We thank Dr. S. T. Cundiff for useful discussions. This work is funded by the NIST and NSF. T. H. Yoon acknowledges financial support from JILA.
[1] Th. Udem et al., Phys. Rev. Lett. 82, 3568 (1999); Th. Udem et al., Opt. Lett. 24, 881 (1999).

[2] S. A. Diddams et al., Phys. Rev. Lett. 84, 5102 (2000).

[3] D. J. Jones et al., Science 288, 635 (2000).

[4] J. E. Bernard et al., Phys. Rev. Lett. 82, 3228 (1999); H. Schnatz et al., ibid. 76, 18 (1996); R. J. Rafac et al., ibid. 85, 2462 (2000); C. W. Oates et al., Opt. Lett. 25, 1603 (2000).

[5] D. Touahri et al., Opt. Commun. 133, 471 (1997).

[6] V. Blanchet et al., Phys. Rev. Lett. 78, 2716 (1997).

[7] M. D. Levenson and N. Bloembergen, Phys. Rev. Lett. 32, 645 (1974).
[8] J. E. Bjorkholm and P. F. Liao, Phys. Rev. Lett. 33, 128 (1974).

[9] O. Poulsen and N. I. Winstrup, Phys. Rev. Lett. 47, 1522 (1981).

[10] E. V. Baklanov and V. P. Chebotaev, Sov. J. Quantum Electron. 7, 1252 (1977); R. Teets et al., Phys. Rev. Lett. 38, 760 (1977).

[11] M. J. Snadden et al., Opt. Commun. 125, 70 (1996).

[12] J. Ye et al., Opt. Lett. 21, 1280 (1996).

[13] R. J. Jones et al., Opt. Commun. 175, 409 (2000).

[14] J. Ye et al., Opt. Lett. 25, 1675 (2000). 